\title{
Lorenzen and Constructive Mathematics
}

\author{
Thierry Coquand
}

\begin{abstract}
The goal of this paper is to present a short survey of some of Lorenzen's contributions to constructive mathematics, and its influence on recent developments in mathematical logic and constructive algebra. We also present some work in measure theory which uses these contributions in an essential way.
\end{abstract}

\section{Introduction}

The school of mathematics in Germany between the two world wars - Noether, Herglotz, Artin, Schmidt, Krull, Hasse, ... - was truly exceptional. This is described in P. Roquette's survey (2018), which emphasizes in particular the importance of the work of Hasse. Lorenzen was Hasse's student, and so was in direct contact with several members of this school.

A new feature was the use of highly non-effective methods in algebra. The axiom of choice was used to show the existence of prime ideals (Krull), or to show the existence of the real or algebraic closure of a given field. A striking example was the use of real algebraic closures by Artin and Schreier (1927) to solve Hilbert's 17 th problem.

Lorenzen was quite unique in this group of mathematicians in being aware of mathematical logic, in particular the contribution of Gentzen. He was able to connect his work in algebra, analysing the use of lattice theory, which started in Dedekind's analysis of ideal numbers, with proof theory. While connections between lattice theory and logic were known since the work of Peirce (1885) and Schröder (1890-1910), connections between lattice

\footnotetext{
Thierry Coquand

Computer Science and Engineering Department, University of Gothenburg, Sweden, e-mail: coquand@chalmers.se
} 
theory and proof theory were quite original. ${ }^{1}$

The work Neuwirth 2020 presents a detailed analysis of this unique situation, containing the following extract of a letter from Krull to Scholz (1953), which illustrates well how Lorenzen's contribution was perceived:

In working with the uncountable, in particular with the well-ordering theorem, I always had the feeling that one uses fictions there that need to be replaced some day by more reasonable concepts. But I was not getting upset over it, because I was convinced that in a careful application of the common "fictions", nothing false comes out, and because I was firmly counting on the man who would some day put all in order. Lorenzen has now found according to my conviction the right way ....

The goal of this paper is to present a short survey of some of Lorenzen's work in constructive mathematics, and its influence on recent developments in mathematical logic and constructive algebra. We also present some work in measure theory which uses Lorenzen's contributions in an essential way.

\section{Lorenzen's analysis of Gentzen's work}

\subsection{The consistency proof}

Lorenzen (1951a) presents Gentzen's consistency proof as a proof about an infinitary cut-free calculus showing that the cut rule is admissible ("zulässig"). Two highly original features of his argument are that the metatheory is constructive (with use of generalised inductive definitions) and that there is no ordinal analysis. At about the same time, and independently, P.S. Novikov (1943) had a similar analysis, and also introduced the notion of an admissible/derivable rule (Citkin 2016). For a historical presentation of Lorenzen's work on infinitary calculus, see Coquand and Neuwirth 2020.

Apart from Novikov, most treatments in proof theory (Gentzen, Schütte, Takeuti) involve ordinal analysis. From a constructive point of view (and for me personally), the purely inductive presentation is much clearer. One objection against this use of infinitary calculus is that, for a consistency proof of arithmetic, the use of generalized inductive definitions is too strong. Indeed, for this purpose the negative translation works just as well, by a purely syntactical argument. Furthermore, an ordinal analysis presents very refined information about what is going on in proofs of arithmetic, information that we cannot get by Lorenzen's proof. However, Lorenzen's argument provides, as we shall see below, an effective description of the free $\sigma$-complete Boolean algebra on a given Boolean algebra, and this is definitely interesting from a mathematical point of view.

${ }^{1}$ The only exception seems to have been the previous work of Skolem (1920). 
For establishing the consistency of stronger calculi, such as $\Pi_{1}^{1}$-analysis, one can even argue that ordinal analysis is, from a constructive point of view, a kind of diversion. For instance, Takeuti proves consistency of this system with a system of ordinal diagrams in a finitary way. To have a constructive explanation of $\Pi_{1}^{1}$-comprehension, however, one needs furthermore, as emphasized, e.g., in Kreisel's (1964) review of Takeuti's proof or in Feferman's (1977) review of Takeuti's book on proof theory, to explain that ordinal diagrams are well-founded in an intuitionistic theory of inductive definitions. A direct explanation of $\Pi_{1}^{1}$-comprehension in an intuitionistic theory of inductive definitions (such as one obtained by use of Buchholz's $\Omega$-rule; Buchholz and Schütte 1988) seems thus to be preferred.

To allow generalized-inductively defined objects in a constructive setting was highly original. Apart from Novikov, the only example of this I could find are proofs in the book Notes on Constructive Mathematics (1968) by Martin-Löf. There, however, infinitary objects are not represented directly, but only via coding as recursively enumerable sets (which arguably obscures the main ideas).

In their paper 1959, Lorenzen and Myhill analyse different ways to define subsets of natural numbers and introduce the stratification given in Table 1.

(I) By explicit definition, quantifying only over natural numbers

(II) By inductive definition, quantifying only over natural numbers

(III) By explicit definition, quantifying only over the (denumerable) totality of sets previously obtained

(Iv) By inductive definition, with the same restriction on quantifiers

(v) By uninhibited use of function-quantifiers

Table 1 Lorenzen-Myhill stratification of definitions of sets.

Use of generalized inductive definitions (Iv) is presented as the "method of Lorenzen" exposed in Lorenzen 1955, with the comment that it "exhausts those means of definition at present known which are acceptable from a standpoint which rejects the actual infinite" (p. 48). The last method (v) is impredicativity, which has no constructive justification. This logical description of methods used in mathematics is quite similar to the one of Martin-Löf in his paper 2008.

The method (Iv) goes beyond what has been called "predicative" mathematics, after the work of Schütte (1965) and Feferman (1964), but it is needed in constructive mathematics, as shown by Lorenzen in his analysis of the Cantor-Bendixson Theorem, which we explain below. 


\subsection{The inversion principle}

In Lorenzen's description of the mathematical universe, we have a calculus of inductively defined objects and inductive proofs/recursively defined functions on these objects.

For instance, we inductively describe the natural numbers by two production rules,

$$
\rightarrow \mid \text { and } x \rightarrow x \mid,
$$

but we also inductively describe the relation of equality, by the two production rules

$$
\rightarrow|=| \quad \text { and } \quad x=y \rightarrow x|=y| .
$$

One important discovery of Lorenzen is the inversion principle (Lorenzen 1955). Let us illustrate this principle on the example of the above inductive description of equality. We have as an admissible rule (this notion was also introduced by Lorenzen)

$$
|=x| \rightarrow \perp,
$$

since there is no way to derive an equality of the form $|=x|$. Similarly, we see that

is an admissible rule.

$$
x|=y| \rightarrow x=y
$$

This way of describing objects and proofs is now common practice in computer science. It is, e.g., used extensively for expressing and proving properties of the semantics of programming languages (as in Kahn's natural semantics; Kahn 1987) in interactive proof systems. Just to give an example, Lorenzen's paper 1951b could almost be written as it is in proof systems for type theory.

In 1992, we noticed that this inversion principle corresponds to the notions of pattern-matching and case notation in functional programming (Coquand 1992). This provides a convenient notation for inductive proofs, which is closely connected to the work Hallnäs and Schroeder-Heister 1990 on definitional reflection. More recent works in this direction are N. Zeilberger's 2009 and J. Cockx' Ph.D. thesis (2017).

\subsection{Distributive lattices and entailment relations}

With respect to this topic, Lorenzen seems to be now mainly known for the following result, which is actually only implicit in his fundamental paper $1951 a$.

Theorem 1 A lattice is distributive if, and only if, it satisfies the (cut) rule

$$
\frac{a \wedge c \leqslant b \quad a \leqslant b \vee c}{a \leqslant b}
$$


This result is cited, e.g., in Curry's book 1976 (Theorem B9, Chap. 4). The paper Lorenzen 1951a actually contains a deeper application of proof theory to the study of distributive lattices, via the notion of an entailment relation. An entailment relation $E, \vdash$ is a relation $a_{1}, \ldots, a_{n} \vdash b_{1}, \ldots, b_{m}$ between finite subsets of a given abstract ${ }^{2}$ set $E$ such that

1. $X \vdash Y$ if $X$ and $Y$ intersect,

2. $X \vdash Y$ if $X^{\prime} \vdash Y^{\prime}$ and $X^{\prime} \subseteq X$ and $Y^{\prime} \subseteq Y$,

3. $X \vdash Y$ if $X, a \vdash Y$ and $X \vdash Y, a$.

We can have $n=0$ or $m=0$, which means that, in this way, we present bounded distributive lattices, i.e., ones with a greatest and a least element. Earlier, Lorenzen (1953) also considered the notion of an unbounded entailment relation, where $m$ and $n$ have to be $>0$.

Entailment relation is the key notion for presenting distributive lattices/ spectral spaces in an elegant way, as explained in Cederquist and Coquand 200o. If $D$ is a (bounded) distributive lattice, an interpretation of $E, \vdash$ is a map $j: E \rightarrow D$ such that $X \vdash Y$ implies $\Lambda j(X) \leqslant \bigvee j(Y)$. By universal algebra, there exists a universal interpretation $i: E \rightarrow L:$ it is an interpretation such that, for any other interpretation $j: E \rightarrow D$, there is a unique map $f: L \rightarrow D$ such that $j=f i$. The following result is essentially stated as such in Lorenzen 1951a. ${ }^{3}$

Theorem 2 (Cederquist and Coquand 2000) Let $E, \vdash$ be an entailment relation. If $L, i: E \rightarrow L$ is the universal interpretation then we have $X \vdash Y$ if, and only if, $\bigwedge i(X) \leqslant \bigvee i(Y)$.

Let us give an example from algebra. On a given integral domain $R$, a valuation for $R$ is a domain $V \supseteq R$ in the field $K$ of fractions of $R$ such that, for any $a \neq 0$ in $K$, we have either $a \in V$ or $a^{-1} \in V$. A fundamental result, proved using Zorn's Lemma, is that an element of $K$ is integral over $R$ (i.e., the root of a unitary polynomial in $R[X])$ if, and only if, it belongs to all valuation domains. Lorenzen was able to describe directly and effectively an unbounded entailment relation $X \vdash Y$ where $X$ and $Y$ are finite sets of nonzero elements of $K$, which, classically, would be equivalent to the following relation: for all valuation domains $V$, there exist $a$ in $X$ and $b$ in $Y$ such that $b / a$ is in $V$.

Lorenzen's (1953) description was the following. If $x_{1}, \ldots, x_{n}$ are elements in the fraction field of $R$, we write $\left(x_{1}, \ldots, x_{n}\right)$ for the $R$-module generated by $x_{1}, \ldots, x_{n}$.

Theorem 3 The relation (for non-zero elements of the field of fractions of $R$ )

$$
a_{1}, \ldots, a_{n} \vdash b_{1}, \ldots, b_{m} \quad \leftrightarrow \quad 1 \in \sum_{i>0}\left(a_{1} b_{1}^{-1}, \ldots, a_{n} b_{m}^{-1}\right)^{i}
$$

\footnotetext{
${ }^{2}$ By this, we mean that $E$ does not need to be a set of syntactically defined objects but can be a set of objects in an arbitrary mathematical structure.

3 There is a similar result for connecting unbounded distributive lattices and unbounded entailment relations.
} 
is an (unbounded) entailment relation which is classically equivalent to the fact that if $V$ is an arbitrary valuation domain then we have $b_{i} / a_{j} \in V$ for some $i, j$.

See Coquand, Lombardi, and Neuwirth 2019 for different proofs and comments on this result. For instance, $a \vdash b$ holds if, and only if, $b$ is integral over $a$. In particular, $b$ is integral over $R$ if, and only if, we have $1 \vdash b$, which can be seen as a constructive version of the result that an element is integral if, and only if, it belongs to all valuation domains. We think this example illustrates well the way Lorenzen's work provides a constructive analysis of non-effective methods in algebra (as evocated in Krull's letter cited in the introduction).

This was in part rediscovered in Coquand and Persson 2001, but expressed there for a bounded entailment relation $X \vdash Y$ on $K$ representing classically the relation: if all elements of $X$ are in $V$ then some element of $Y$ is in $V$.

\section{Proof-theoretic analysis of point-free spaces}

In this section, we want to present Lorenzen's (1958) analysis of CantorBendixson's Theorem. It states that if $F$ is a closed subset of $[0,1]$ then we can find a closed subset $K \subseteq F$ which is perfect (i.e., $K$ has no isolated points) and such that $F-K$ is countable. Since $K$ has the power of the continuum if it is not empty, this shows that the continuum hypothesis holds for closed subsets of $[0,1]$. The way we build $K$ is by a transfinite process: we first define the derivative $F^{\prime}$ of $F$ obtained by removing from $F$ its isolated point, and we iterate this operation (maybe transfinitely) in order to get a fixed point $K=K^{\prime}$. The analysis of this theorem was crucially needed in Kreisel's paper 1959. Defining the kernel requires, a priori, being at stage (v) of the LorenzenMyhill stratification in Table 1 . What is remarkable about this result is that, as shown by Kreisel, Cantor-Bendixson's Theorem requires methods going beyond what has been called "predicative mathematics" by Feferman (1977) and Schütte (1965), but, and this is Lorenzen's contribution, it can be captured constructively using Method (IV) of Table 1. In characteristic manner, Lorenzen presents in his 1958 only the main idea, without ever providing all the details (they can be found in the proof of Theorem 1 of Kreisel 1959).

In order to present this analysis as simply as possible, we will do it for Cantor space instead of $[0,1]$ (as is done in Lorenzen 1958). As a set of points, the Cantor space is the set $\Omega$ of infinite binary sequences $\omega=\omega_{0}, \omega_{1}, \omega_{2}, \ldots$ As a point-free space, where we describe directly in algebraic (and effective) terms its compact open subsets, it can be seen as the Boolean algebra $C$ of propositional logic, i.e., the Boolean algebra freely generated by countably many formal atoms written $\omega_{k}=1$ or $\omega_{k}=0$. For instance, $\omega_{1}=0 \wedge \omega_{3}=1$ represents a compact open subset of $\Omega$, namely all sequences $\omega$ such that $\omega_{1}=0$ and $\omega_{3}=1$. We write $\sigma_{,} \sigma_{1}$, etc. for finite binary sequences. Each such 
finite sequence $\sigma$ represents a compact open subset, namely the set of all infinite sequences extending $\sigma$. Seen as a set of sequences, $\sigma$ is equal to the union of the two sets defined by its direct extensions $\sigma 0$ and $\sigma 1$.

Using this representation, an open subset $U$ of $\Omega$ can be represented as a predicate $U(\sigma)$ such that $U(\sigma)$ holds if, and only if, we have both $U(\sigma 0)$ and $U(\sigma 1)$. In terms of sets of infinite sequences, $U(\sigma)$ expresses that the compact open set represented by $\sigma$ is a subset of the open set represented by $U$. Dually, such a predicate can also be thought of as representing the closed subset complement $F=\Omega \backslash U$. As explained above, the main operation in Cantor-Bendixson's Theorem is the forming of the derivative of a closed subset, which is obtained by taking away the isolated points of this subset. Dually, this can be understood as an operation $U^{\prime} \supseteq U$ on open subsets $U$ such that $\Omega \backslash U^{\prime}$ is the derivative of $\Omega \backslash U$.

One crucial insight of Lorenzen is that we can define $U^{\prime}$ in terms of an operation on predicates on finite binary sequences. We can indeed express the fact that $\sigma$ contains at most one point of $\Omega \backslash U$ as follows: at level 1, we have $U(\sigma 0)$ or $U(\sigma 1)$, at level 2, we have $U(\sigma 0) \wedge U(\sigma 10)$ or $U(\sigma 0) \wedge$ $U(\sigma 11)$ or $U(\sigma 1) \wedge U(\sigma 00)$ or $U(\sigma 1) \wedge U(\sigma 01)$, and so on. We can thus write a formula $A(U, \sigma)$ involving a universal quantification on natural numbers, such that $A(U, \sigma)$ expresses that $\sigma$, seen as a set of infinite sequences, contains at most one point in $\Omega \backslash U$. The inductive definition of $U^{\prime}$ is then
1. $U^{\prime}(\sigma 0) \wedge U^{\prime}(\sigma 1) \rightarrow U^{\prime}(\sigma)$,
2. $A(U, \sigma) \rightarrow U^{\prime}(\sigma)$,

and $U^{\prime}(\sigma)$ expresses classically that there are only finitely many points of $F$ in the compact open set represented by $\sigma$.

The kernel of $F$ is obtained by iterating (maybe transfinitely) the derivative operation for $F$. In terms of open sets, this can be represented by the following inductive definition:

$$
\begin{aligned}
& \text { 1. } U(\sigma) \rightarrow V(\sigma), \\
& \text { 2. } V(\sigma 0) \wedge V(\sigma 1) \rightarrow V(\sigma), \\
& \text { 3. } A(V, \sigma) \rightarrow V(\sigma) .
\end{aligned}
$$

With this definition, we have that $\Omega \backslash V$ represents the kernel of $\Omega \backslash U$. Since the predicate $A(V, \sigma)$ involves a universal quantification on natural numbers, this description of the kernel uses a generalized inductive definition (Lorenzen and Myhill 1959), but it is constructive and does not involve a classical theory of uncountable ordinals. 


\section{Measure theory}

\subsection{Borel subsets of Cantor space}

The analysis by Lorenzen of Gentzen's cut-elimination contains an effective description the $\sigma$-complete Boolean algebra generated by a given Boolean algebra. More generally, given an entailment relation $E, \vdash$ as defined above, Lorenzen describes the $\sigma$-complete Boolean algebra $B$ with an interpretation $v: E \rightarrow B$ universal for this property. He then proves

Theorem 4 For the universal $\sigma$-complete Boolean algebra $B$ with an interpretation v: $E \rightarrow B$, we have

$$
a_{1}, \ldots, a_{n} \vdash b_{1}, \ldots, b_{m} \quad \leftrightarrow \quad v\left(a_{1}\right) \wedge \cdots \wedge v\left(a_{n}\right) \leqslant v\left(b_{1}\right) \vee \cdots \vee v\left(b_{m}\right) .
$$

This result is cited in the reference Beth 1959 (which might, surprisingly, be the only published reference to this remarkable result from the paper Lorenzen 1951a). If we start from the Boolean algebra $C$ of propositional logic, which is the Boolean algebra generated from countably many atoms, we get a $\sigma$-complete Boolean algebra $B$. As explained above, $C$ can be seen as a point-free presentation of Cantor space, which is the set $\Omega$ of all infinite binary sequences $\omega=\omega_{0}, \omega_{1}, \ldots$ The algebra $B$ can then in turn be seen as a point-free presentation of the $\sigma$-complete Boolean algebra of Borel sets on Cantor space. This was noticed by Martin-Löf (1968). If we start from the Boolean algebra with two elements, we get the $\sigma$-complete Boolean algebra of hyperarithmetical propositions.

In this point-free view, a Borel set $X$ is given inductively: $X$ is a propositional formula or $X$ is of the form $\bigvee_{n} X_{n}$ or $X$ is of the form $\wedge_{n} X_{n}$. Lorenzen defines a sequent calculus $X_{1}, \ldots, X_{n} \vdash Y_{1}, \ldots, Y_{m}$ and proves that the cut rule is admissible. This means that we can prove the rule

$$
\frac{\Gamma, X \vdash \Delta \quad \Gamma \vdash X, \Delta}{\Gamma \vdash \Delta}
$$

by case analysis and induction on the two given derivations. Essentially the same argument is done in Martin-Löf 1968.

We can define $X \subseteq Y$ by $X \vdash Y$. We have $X \subseteq X$ by induction on $X$ and, using cut-elimination, $X \subseteq Z$ if $X \subseteq Y$ and $Y \subseteq Z$. Indeed, if we have $X \vdash Y$ and $Y \vdash Z$ then by weakening we get $X \vdash Y, Z$ and $X, Y \vdash Z$, and then by cut-elimination we get $X \vdash Z$.

An example of a point-free description is the set of normal binary sequences

$$
N=\bigwedge_{k} \bigvee_{m} \bigwedge_{n \geqslant m} b_{n, k}
$$

with $b_{n, k}$ a point-free representation of 


$$
\left\{\omega \in \Omega \mid-\frac{1}{k} \leqslant \frac{\sum_{i<n}\left(2 \omega_{i}-1\right)}{n} \leqslant \frac{1}{k}\right\} .
$$

In the classical approach this is thought of as a set of points (the complement of which is not countable and of measure 0). In the present setting, it is a purely symbolic expression. A satisfactory theory of the measures of Borel sets should prove that this set, defined in this "symbolic" way, is of measure 1.

\subsection{Borel's measure problem}

As explained above, Borel sets can be described inductively. The following is then a natural question: Can we define the measure $\mu(X)$ of a Borel set $X$ by induction on $X$ ? Borel's (1894) own formulation was the following (for subsets of $[0,1]$ ): we design a formal theory which describes how the measure should work, and we have to prove that this formal theory is consistent.

As presented by Lusin (1930), the question can be seen as a coherence problem: we have to provide an inductive definition of the measure $\mu(X)$ of a Borel set $X$ such that $X \vdash Y \rightarrow \mu(X) \leqslant \mu(Y)$. Lusin, in his book 1930, asked for a purely inductive solution of this problem, and called this question Borel's measure problem.

\subsection{An inductive solution of Borel's measure problem}

In 1959 (p. 48), Lorenzen and Myhill wrote, referring to the stratification in Table 1:

We regard as important the problem of determining what sets, relative to the function-quantifier hierarchy, are definable by this method. For Method (Iv) exhausts those means of definition at present known which are acceptable from a standpoint which rejects the actual infinite; so that the determination of the extent to which sets definable by this method penetrate the functionquantifier hierarchy would be of no small significance from the point of view of foundations. For it would yield a quantitative measure of the extent to which discourse involving the quantifiers $\Lambda_{f}$ and $\bigvee_{f}$, which seems to refer to an actual infinite, can be so paraphrased as to avoid such statements.

We provide here such an analysis for measure theory. In the usual treatment (Lebesgue, Daniell, Bourbaki), it refers to an actual infinite. It can, however, as we explain here, be described purely at the level of Method (IV), i.e., using generalized inductive definitions.

Here, I show how to recursively define $r<\mu(X)$ as a hyperarithmetical proposition by induction on $X$. We take the usual measure on Cantor space: 
if $X$ is a propositional formula, $\mu(X)$ is a rational and $r<\mu(X)$ is 0 or 1 . For instance, $\mu\left(\omega_{1}=0 \wedge \omega_{3}=1\right)=1 / 4$.

The approach of Borel (1894), inspired by the work of Drach, is to specify abstractly what properties any measure should satisfy, and to prove then that these conditions do not produce any contradiction. The condition is that the measure of a disjoint countable union be the sum of the measures (and to require in particular that this sum actually converges).

Here is a simple example of the potential coherence problem in this specification. If we define $X_{0}=\bigwedge_{k}\left[\omega_{k}=0\right]$ and $X_{n+1}=\bigwedge_{i<n}\left[\omega_{i}=0\right] \wedge\left[\omega_{n}=1\right]$, we have $1=\bigvee_{n} X_{n}$ and $\mu(1)=1$ and $\mu\left(X_{0}\right)=0$ and $\mu\left(X_{n+1}\right)=1 / 2^{n+1}$. We see that we have two ways to write 1 as a disjoint sum of elements: either as 1 itself or as the disjoint sum of the $X_{n}$. We can then check the consistency of these two possible ways to compute $\mu(1)$ since we have $1=0+1 / 2+1 / 4+\cdots$.

The main difficulty in this inductive approach is how to define $r<\mu(X)$ if $X$ is a disjunction or conjunction. The problem is that if $X$ is, for instance, a disjunction $\bigvee_{n} X_{n}$, not necessarily disjoint, then $\mu(X)$ is not a function of the sequence $\mu\left(X_{n}\right)$ anymore. There is thus a problem in defining $\mu(X)$ by induction on $X$.

One solution is provided by the remarkable paper of F. Riesz (1930): if $X$ is the disjunction of the family $X_{n}$ then the function $\mu_{X}: b \mapsto \mu(b \wedge X)$ on propositional formulas can be defined in terms of $\mu_{X_{n}}$.

The recursive definition of F. Riesz is, for $X=\bigvee_{n} X_{n}$, given by

$$
\mu_{X}(b)=\bigvee_{\substack{b=b_{1}, \ldots, b_{k} \\ n_{1}<\ldots<n_{k}}} \sum_{k=1}^{k} \mu_{X_{n_{k}}}\left(b_{\kappa}\right),
$$

where $b=b_{1}, \ldots, b_{k}$ is a partition of $b$.

If $X=c$ then we can compute $r<\mu(b \wedge c)$, and this is the value of $r<\mu(b \wedge X)$.

If $X=\bigvee_{n} X_{n}$ then $r<\mu(b \wedge X)$ is the formula

$$
\bigvee_{\substack{b=b_{1}, \ldots, b_{k} \\ r=b_{1}+\cdots, r_{k} \\ n_{1}<\cdots<n_{k}}} \bigwedge_{\kappa=1}^{k}\left(r_{\kappa}<\mu\left(b_{\kappa} \wedge X_{n_{\kappa}}\right)\right) .
$$

For $X=\bigwedge_{n} X_{n}$, we should have $\mu(b \wedge X)=\mu(b)-\mu\left(b \wedge \bigvee_{n} X_{n}^{\prime}\right)$, where $X_{n}^{\prime}$ is the formal complement of $X_{n}$ and

$$
\mu\left(b \wedge \bigvee_{n} X_{n}^{\prime}\right)=\bigvee_{\substack{b=b_{1}, \ldots, b_{k} \\ n_{1}<\cdots<n_{k}}} \sum_{\kappa=1}^{k} \mu\left(b_{\kappa} \wedge X_{n_{\kappa}}^{\prime}\right)
$$

From this, we deduce the value of $r<\mu(b \wedge X)$ as the formula 


$$
\bigvee_{r<s} \bigwedge_{\substack{b=b_{1}, \ldots, b_{k} \\ n_{1}<\cdots<n_{k}}} \bigvee_{s=s_{1}+\cdots+s_{k}} \bigwedge_{\kappa=1}^{k} s_{\kappa}<\mu\left(b_{\kappa} \wedge X_{n_{\kappa}}\right) .
$$

Using these equations recursively, we define $r<\mu(b \wedge X)$ as a hyperarithmetical formula.

In this way, we get a purely inductive description of measure theory. We are at stage (IV) of Lorenzen and Myhill's stratification in Table 1. The proof given by Riesz (and by Lebesgue, Daniell, Bourbaki) that the definitions work, however, uses impredicative arguments that are at stage (v) of this stratification. The new discovery is that it is possible to show in a purely inductive way the following result.

Theorem 5 If $X \vdash Y$ then $[r<\mu(b \wedge X)] \leqslant[r<\mu(b \wedge Y)]$. Hence if $X$ and $Y$ define the same Borel subset of Cantor space, $\mu(X)=\mu(Y)$.

This shows the consistency of our definition: if $X$ and $Y$ represent the same Borel set then $r<\mu(b \wedge X)$ and $r<\mu(b \wedge Y)$ are equal.

This analysis is provided in Coquand 2004, where I also give a presentation of measure theory using only generalized inductive definitions and no impredicative arguments.

As an application, we can show, purely inductively, that $r<\mu(1 \wedge N)$ is provable for each $r<1$, where $N$ is the symbolic representation of the set of normal binary sequences described above. We get in this way a proof of $\mu(N)=1$ which involves only inductive reasoning.

\section{Game semantics}

In this last section, I briefly present Lorenzen's extremely original work on game semantics. A formula is seen as specifying a game between two players, the proponent and the opponent, who argue about the truth value of the formula. A proof of the formula is then seen as a winning strategy for the proponent in this game. Lorenzen (1959) has, for instance, a suggestive analysis of the formula $\neg \neg a \rightarrow a$ and of why it is not intuitionistically valid. The idea is to consider a statement $a$ for which the opponent has a proof which is not known by the proponent. If the opponent asserts $\neg \neg a$, the proponent (who does not know the proof of $a$ ) has to challenge the opponent by asserting $\neg a$ (hoping that the opponent does not know the proof of $a$ either). But then the opponent wins by giving the proof of $a$.

Lorenzen (1959) mentions that we can get an interpretation of classical logic by allowing the proponent to backtrack. In Coquand 1995, I suggested an analysis of cut-elimination based on this interpretation, describing cutelimination as an interaction between two strategies that can both backtrack. We can in this way give a proof of termination of the cut-elimination process, 
essentially different from the one of Gentzen. This has recently been used by F. Aschieri (2017) to provide a nontrivial refinement of Gentzen's upper bound (with a tower of exponentials) for the depth of the resulting proof obtained by the cut-elimination process in terms of the level of backtracking of the strategies. For instance, if one strategy has only one level of backtracking, the upper bound is given by a single exponential (whatever the complexity of the cut formula).

This idea of game interpretation has also been refined in various ways. An extension of this interpretation to analysis is described in Berardi, Bezem, and Coquand 1995, giving in particular a different interpretation than the one of Spector (1961). See also the interpretation of the axiom of determinacy in Hida 2012.

\section{References}

Artin, Emil, and Otto Schreier. 1927. "Eine Kennzeichnung der reell abgeschlossenen Körper." Abhandlungen aus dem Mathematischen Seminar der Universität Hamburg 5:225-231.

Aschieri, Federico. 2017. "Game semantics and the geometry of backtracking: A new complexity analysis of interaction." Journal of Symbolic Logic 82 (2): $672-708$.

Berardi, Stefano, Marc Bezem, and Thierry Coquand. 1995. "A realization of the negative interpretation of the Axiom of Choice." In Typed lambda calculi and applications: Second international conference on typed lambda calculi and applications, TLCA'95, Edinburgh, UK, April 10-12, 1995, Proceedings, edited by M. Dezani-Ciancaglini and G. D. Plotkin, pages 47-62. Lecture Notes in Computer Science, volume 902. Springer. doi:10.1007/BFb0014044

Beth, Evert Willem. 1959. The foundations of mathematics: A study in the philosophy of science. Amsterdam: North-Holland.

Borel, Émile. 1894. Sur quelques points de la théorie des fonctions. GauthierVillars.

Buchholz, Wilfried, and Kurt Schütte. 1988. Proof theory of impredicative subsystems of analysis. Naples: Bibliopolis.

Cederquist, Jan, and Thierry Coquand. 2000. "Entailment relations and distributive lattices." In Logic colloquium '98, edited by S. R. Buss, P. Hájek, and P. Pudlák, pages 127-139. Association for Symbolic Logic.

Citkin, Alex. 2016. "Multiple conclusion rules in logics with the disjunction property." In Logical Foundations of Computer Science: LFCS 2016, edited by S. Artemov and A. Nerode, pages 76-89. Lecture Notes in Computer Science, volume 9537. Cham: Springer.

Cockx, Jesper. 2017. Dependent pattern matching and proof-relevant unification. Ph.D. thesis, KU Leuven. 
Coquand, Thierry. 1992. "Pattern matching with dependent types." Informal proceedings of Logical Frameworks, pages 66-79. doi: 10.1.1.37.9541

_. 1995. "A semantics of evidence for classical arithmetic." Journal of Symbolic Logic 6o (1): 325-337.

- 2004. "A note on measures with values in a partially ordered vector space." Positivity 8 (4): 395-400.

Coquand, Thierry, Henri Lombardi, and Stefan Neuwirth. 2019. "Latticeordered groups generated by an ordered group and regular systems of ideals." Rocky Mountain Journal of Mathematics 49:1449-1489. doi: 10.1216/rmj-2019-49-5-1449.

Coquand, Thierry, and Stefan Neuwirth. 2020. "Lorenzen's proof of consistency for elementary number theory." History and Philosophy of Logic, to appear. doi:10.1080/01445340.2020.1752034

Coquand, Thierry, and Henrik Persson. 2001. "Valuations and Dedekind's Prague theorem." Journal of Pure and Applied Algebra 155 (2-3): 121-129.

Curry, Haskell B. 1976. Foundations of mathematical logic. Dover.

Feferman, Solomon. 1964. "Systems of predicative analysis." Journal of Symbolic Logic 29:1-30.

. 1977. Review of Proof theory by G. Takeuti. Bulletin of the American Mathematical Society 83 (3): 351-361.

Hallnäs, Lars, and Peter Schroeder-Heister. 1990. "A proof-theoretic approach to logic programming. I. Clauses as rules." Journal of Logic and Computation 1 (2): 261-283.

Hida, Takanori. 2012. "A computational interpretation of the axiom of determinacy in arithmetic." In Computer Science Logic (CSL'12) - 26th International Workshop/21st Annual Conference of the EACSL, edited by P. Cégielski and A. Durand, pages 335-349. Schloss Dagstuhl-LeibnizZentrum für Informatik.

Johnstone, Peter T. 1982. Stone spaces. Cambridge studies in advanced mathematics 3. Cambridge: Cambridge University Press.

Kahn, G. 1987. "Natural semantics." In STACS 87: 4th Annual Symposium on Theoretical Aspects of Computer Science: Passau, Federal Republic of Germany, February 19-21, 1987: Proceedings, pages 22-39. Berlin/Heidelberg: Springer.

Kreisel, Georg. 1959. "Analysis of Cantor-Bendixson theorem by means of the analytic hierarchy." Bulletin de l'Académie Polonaise des Sciences 7:621-626.

- 1964. Review of On the fundamental conjecture of GLC. vi by G. Takeuti. Zentralblatt für Mathematik und ihre Grenzgebiete 106 (2): 237-238.

Lorenzen, Paul. 1951a. "Algebraische und logistische Untersuchungen über freie Verbände." Journal of Symbolic Logic 16:81-106. URL: http: //www.jstor.org/stable/2266681. Translation by S. Neuwirth: Algebraic and logistic investigations on free lattices, URL: http://arxi 
v.org/abs/1710.08138. 338.

. 1951b. "Über endliche Mengen." Mathematische Annalen 123:331-

. gruppen." Mathematische Zeitschrift 58:15-24.

- 1955. Einführung in die operative Logik und Mathematik. Berlin.

. 1958. "Logical reflection and formalism." Journal of Symbolic Logic 23 (3): $241-249$.

. 1959. "Operative mathematics and computers." In Computer programming and artificial intelligence: An intensive course for practicing scientists and engineers: Lectures given at the University of Michigan, summer 1958, edited by J.W. Carr, pages 405-426. College of Engineering, University of Michigan.

Lorenzen, Paul, and John Myhill. 1959. "Constructive definition of certain analytic sets of numbers." Journal of Symbolic Logic 24 (1): 37-49.

Lusin, Nicolas. 1930. Leçons sur les ensembles analytiques et leurs applications. Paris: Gauthier-Villars.

Martin-Löf, Per. 1968. Notes on constructive mathematics. Stockholm: Almqvist \& Wiksell.

- 2008. "The Hilbert-Brouwer controversy resolved?" In One hundred years of intuitionism (1907-2007): The Cerisy conference, edited by M. van Atten, P. Boldini, M. Bourdeau, and G. Heinzmann, pages 243-256. Basel/Boston/Berlin: Birkhäuser.

Neuwirth, Stefan. 2020. "Lorenzen's reshaping of Krull's Fundamentalsatz for integral domains (1938-1953)." In this volume, pages 141-180.

Novikov, Pyotr Sergeyevich. 1943. "On the consistency of a certain logical calculus." Matematičeskij sbornik/Recueil mathématique 12 (54): 230-260.

Peirce, Charles Sanders. 1885. "On the algebra of logic: A contribution to the philosophy of notation." American Journal of Mathematics 7 (2): 180-202.

Riesz, Frédéric. 1930. "Sur la décomposition des opérations fonctionnelles linéaires." In Atti del Congresso Internazionale dei Matematici, Bologna 1928, volume 3, pages 143-148.

Roquette, Peter. 2018. The Riemann hypothesis in characteristic $p$ in historical perspective. Cham: Springer.

Schröder, Ernst. 1890-1910. Vorlesungen über die Algebra der Logik, volumes I-III. Reprint Chelsea 1966.

Schütte, Kurt. 1965. "Predicative well-orderings." In Formal systems and recursive functions: Proceedings of the eighth logic colloquium, Oxford, July 1963 (volume 40 of Studies in Logic and the Foundations of Mathematics), edited by J. N. Crossley and M. A. E. Dummett, pages 280-303. Elsevier.

Skolem, Thoralf. 1920. "Logisch-kombinatorische Untersuchungen über die Erfüllbarkeit oder Beweisbarkeit mathematischer Sätze nebst einem Theoreme über dichte Mengen." Videnskapsselskapets skrifter, I. Matematisk-naturvidenskabelig klasse 4:1-36. 
Spector, Clifford. 1961. "Provably recursive functionals of analysis: A consistency proof of analysis by an extension of principles formulated in current intuitionistic mathematics." In Recursive function theory: Proceedings of symposia in pure mathematics, volume v, pages 1-27. Providence, RI: American Mathematical Society.

Zeilberger, Noam. 2009. The logical basis of evaluation order and patternmatching. Ph.D. thesis, Carnegie-Mellon University.

Open Access This chapter is licensed under the terms of the Creative Commons Attribution 4.0 International License (http://creativecommons.org/licenses/by/4.0/), which permits use, sharing, adaptation, distribution and reproduction in any medium or format, as long as you give appropriate credit to the original author(s) and the source, provide a link to the Creative Commons license and indicate if changes were made.

The images or other third party material in this chapter are included in the chapter's Creative Commons license, unless indicated otherwise in a credit line to the material. If material is not included in the chapter's Creative Commons license and your intended use is not permitted by statutory regulation or exceeds the permitted use, you will need to obtain permission directly from the copyright holder.

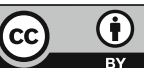

